\title{
Diagnostic Value of Multiplex PCR in Acute Bacterial Meningitis; Single Center Experience
}

E. Kepenekli Kadayifci1, P. Cennetoğlu2 G. Soyletir3, R. Can Sarinoglu3, O.M. Kumbul2.

1:Marmara University School of Medicine, Pendik Training and Research Hospital, Division of Pediatric Infectious Diseases, Istanbul, Turkey.

2: Marmara University School of Medicine, Pendik Training and Research Hospital, Department of Pediatrics, Istanbul, Turkey.

3: Marmara University School of Medicine, Pendik Training and Research Hospital, Department of Medical Microbiology, Istanbul, Turkey.

Background

Acute bacterial meningitis is an important cause of morbidity and mortality in children. The definition of meningitis is inflammation of pia and arachnoid membranes that cover the brain and spinal cord. Clinical signs and symptoms include sudden onset of fever, headache, stiff neck, nausea, vomiting and altered mental status. Signs and symptoms may occur within a few hours to several days. Acute bacterial meningitis should be promptly diagnosed and the appropriate therapy should be initated early to prevent it's complications such as convulsion, ventriculitis, hydrocephalus, subdural effusion and brain abscess. Meningitis can be diagnosed based on a medical history, physical examination, laboratory tests including complete blood count, acute phase reactant and blood culture. The most important diagnostic test is the evaluation of the cerebrospinal fluid (CSF) for cell count and protein and glucose levels. CSF gram staining, culture and multiplex PCR analysis can define the causative agent. Neuroimaging also can be used for diagnosis.

Causative agents may vary by age, underlying disease and geographical area. The most common pathogens are; Streptococcus Pneumoniae, Neisseria meningitidis, Haemophilus influenzae type $b$. Despite of cerebrospinal fluid culture is accepted gold standard for diagnosis; it can resulted as negative esspecially in patient with previous antibiotic usage in history. Multiplex PCR ( Biofire FilmArray ${ }^{\circledR}$ ), ensures important advantages at diagnosis and timely treatment by resulting within first 1 hour of CSF examination and resulting positive despite of negative CSF culture due to previous antibiotic usage.

Methods

Multiplex PCR (Biofire FilmArray ${ }^{\circledR}$ ), meningitis/encephalitis panel tests 14 pathogens that include Escherichia coli K1, Haemophilus influenzae, Listeria monocytogenes, Neisseria meningitidis, Streptococcus pneumoniae, Cytomegalovirus, Enterovirus, Herpes simplex virus 1, Herpes simplex virus 2, Human herpes virus 6, Human parechovirus, Varicella zoster virus, Cryptococcus neoformans/gattii and it is resulted in 1 hour.

In this study, we evaluated the value of multiplex PCR on diagnosis and treatment of acute bacterial meningitis, in patients between $0-18$ years of age with the presumed diagnonis of meningitis who admitted to Marmara University Pendik Training and Research Hospital. Biofire Filmarray Multiplex PCR was performed in CSF samples of 59 patients and compared with CSF culture results.

Lumbar puncture was performed in patients with clinical signs or symptoms of meningitis sucs as fever, seizure, vomitting, rash, head ache, meningeal irritation findings, pulsatile fontanel, changes in consciousness. Glucose and protein levels, bacterial culture results, leucocyte count and multiplex PCR results were recorded.

\section{Results}

Multiplex PCR resulted as positive in 12 of 59 patients, while CSF bacterial culture resulted as positive in 4 of them. Viral pathogens were detected in 7 patients.

Neisseria meningitidis was detected in 3 patients with multiplex PCR in first hour of admission. The CSF culture was sterile in 1 of 3 patients. The antibiotic treatment, isolation precautions and postexposure prophylaxis for contacts performed rapidly.

Streptococcus pneumoniae was detected in 2 patients with multiplex PCR test while thei bacterial cultures were sterile.

Viral pathogens were detected 7 patients and antibiotic treatment was stopped early in management ( Table 1).

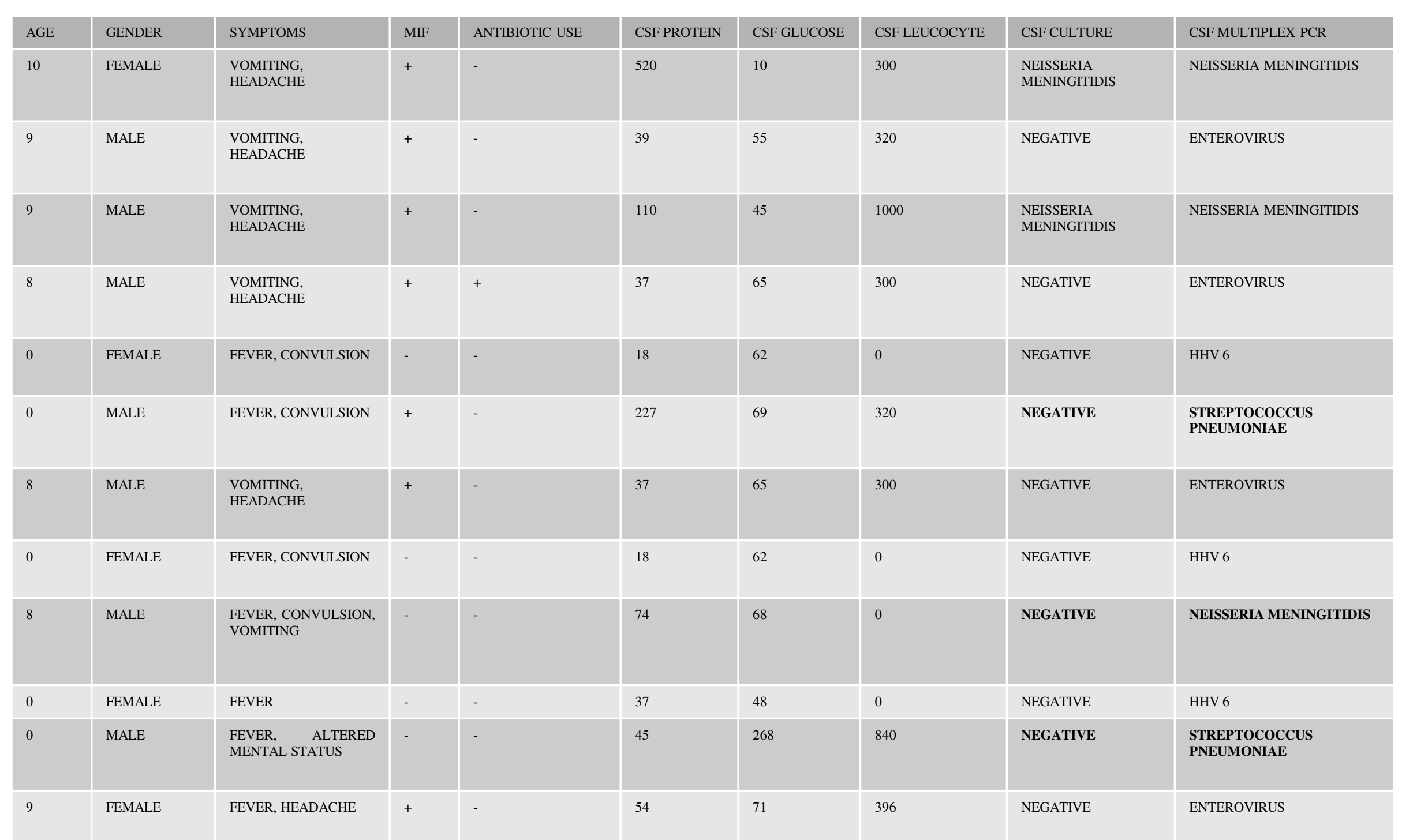

\section{Discussion}

When the studies were evaulated, it has been shown that Multiplex PCR (Biofire FilmArray ${ }^{\circledR}$ ), ensures important advantages in diagnosis and treatment due to it's quick resulting time $(1,2)$. In bacterial meningitis cases, one of the important reason for sterile culture is the antibitoic usage prior to diagnosis. It has been shown that multiplex PCR may result positive while culture is sterile (3). Early initiation of appropriate treatment is provided by rapid positive results also negative results can prevent unnecessary antibiotic therapy (4)

\section{Conclusion}

Acute bacterial meningitis is an important cause of morbidity and mortality in children. Multiplex PCR (Biofire FilmArray ${ }^{\circledR}$ ), ensures important advantages at diagnosis and treatment by resulting rapidly, testing many pathogens at once and also by resulting positive while culture is sterile. 